\title{
Heart rate variability in trained athletes and sedentary individuals- A Comparative study
}

\author{
Vedashree Hegde $^{1}$, N G Borade ${ }^{2, *}$, S. Naveen ${ }^{3}$, Bhargava V. Devarakonda ${ }^{4}$ \\ ${ }^{1}$ PG Student, ${ }^{2}$ Professor \& HOD, Dept. of Physiology, Dr. D. Y. Patil Medical College and Research Centre, Pimpri, Pune, \\ Maharashtra, ${ }^{3}$ Senior Advisor, ${ }^{4}$ Graded Specialist, Dept. of Anaesthesia, Military Hospital, Kirkee, Pune, Maharashtra, India
}

\section{*Corresponding Author:}

Email:ngborade@gmail.com

Received: $12^{\text {th }}$ February, 2018

Accepted: $19^{\text {th }}$ February, 2018

\begin{abstract}
Introduction: Sedentary lifestyle has given rise to various lifestyle diseases. Regular exercise training is known to improve cardiovascular functions. Heart rate variability analysis is done to assess cardiac autonomic function. The present study is to assess and compare heart rate variability in trained athletes and sedentary individuals.

Materials and Methods: A cross sectional study, matched for age and sex, was done in 30 apparently healthy trained athletes and 30 apparently healthy sedentary individuals. BMI, resting pulse, blood pressure and heart rate variability analysis was done and analysed statistically.

Results: In trained athletes; parameters of heart rate variability RMSSD (Root Mean Sum of Square Difference), Low Frequency (LF) and High Frequency (HF) showed significant increase and Resting pulse rate and Diastolic blood pressure showed significant decrease as compared to sedentary individuals.

Conclusion: Heart rate variability is increased in trained athletes than sedentary individuals.
\end{abstract}

Keywords: Heart rate variability, Trained Athletes, Sedentary individuals.

\section{Introduction}

In this era of mechanization with mobiles, computers, television and various electrical and electronic gadgets, there has been a drastic decrease in physical activity at home as well as work place. Many of the office goers have to sit for prolonged periods while commuting or at work place. Even children spend most of their time indoors with less physical activity in the school or at home. ${ }^{1}$ Sedentary lifestyle has given rise to a huge problem of lifestyle diseases which are affecting our day to day living and work. Medical research to pinpoint exact reasons for these diseases are being conducted so as to prevent and cure them.

Pursuing physical activities like sports, exercises such as walking, jogging, swimming or yoga can help in achieving efficient cardio respiratory functions and is thus helpful in prevention of lifestyle diseases. Specifically, it has been found that exercises alter cardiac autonomic functions of the body. ${ }^{2}$

Autonomic nervous system plays pivotal role in the regulation of cardiovascular activities. ${ }^{3}$ The autonomic functions of the heart can be measured by Heart Rate Variability. Heart rate variability is beat to beat variability of heart rate. Decrease in Heart Rate Variability indicates autonomic dysfunction. Heart Rate Variability also has prognostic significance for cardiovascular diseases. ${ }^{4}$

Several studies have been conducted previously to know the modulations in autonomic functions due to exercise. It is documented that athletes have resting bradycardia. However sympatho-vagal balance is by moderation of sympathetic system or by enhancement of vagal tone is not very clear.

Also, long duration of training by athletes has not been studied extensively to know long term effects of athletic training on individuals. Hence the present study, to assess and compare the Heart Rate Variability in trained athletes and sedentary individuals.

\section{Materials and Methods}

Institutional Ethics Committee (IEC) clearance was obtained before commencement of study. The period of study was from July 2015 to September 2017. A cross sectional study was conducted. A total of 60 subjects taken as sample size based on confidence interval. They were selected in the age group of 20 to 45 years. Group I - Consisted of 30 apparently healthy trained athletes who have regularly run for at least the past 6 months. Regular practice was defined as running $5 \mathrm{Kms}$ in 30 minutes at a speed of $10 \mathrm{kmph}$ for a minimum of three sessions per week. ${ }^{1,5}$ Running could take place in any setting (indoor or outdoor) under supervision of a trainer. Group II - Consisted of 30 apparently healthy individuals of sedentary lifestyle who carried out only their daily chores and did not do any form of extra physical exercise to improve their physical fitness. This non exercise regimen was continued for six months. ${ }^{6}$ Individuals with any history of substance abuse like smoking, consumption of alcohol or a history of any known chronic systemic disease were excluded. All subjects were explained in detail about the purpose and methodology of the study. Written informed consent 
was obtained from each subject. Detailed medical, family, occupational, personal, drug history of all the subjects was taken. Duration of the exercise practice sessions was noted for Group I subjects. To maintain constancy of conditions, all the tests were carried out between 10 AM and 1 PM. It is known that circadian rhythm causes changes in ANS balance during different times of the day and may alter measurements. ${ }^{16}$

\section{Procedure}

Body Mass Index (BMI) was calculated by standard formula weight in kilograms divided by height in square of centimetres. ${ }^{7}$

Resting pulse rate and resting blood pressure ${ }^{8}$ were recorded.

Heart Rate Variability (HRV) at rest ${ }^{4}$ was recorded using RMS Polyrite D (multichannel digitalized polygraph machine, model version 2.4, Recorders and Medicare Systems Private Limited, Chandigarh, India). After a complete rest in supine position for 15 minutes, baseline acquisition was recorded in ECG mode on Polyrite D machine. Continuous ECG (lead II) was recorded at $30 \mathrm{~mm} / \mathrm{sec}$ for 5 minutes in supine position with quiet breathing. Stabilized signals were stored after automatically filtering ectopic beats, artifacts, noise. Recording was analysed with inbuilt software and HRV parameters were assessed in Time Domain and Frequency Domain as Spectral Density analysis by Fast Fourier Transformation (FFT).

Time Domain measured are: a) SDNN (Standard Deviation of NN intervals that is, square root of their deviation). b) RMSSD (Square Root of the Mean Squared Differences of Successive NN intervals).

Frequency Domain Methods: The components of spectrum that are mainly described are i) very low frequency (VLF) ii) low frequency (LF) iii) high frequency (HF) components. VLF, LF and $\mathrm{HF}$ components are usually measured in absolute values of power $\left(\mathrm{ms}^{2}\right)$, but normalized units (n.u.) can be used to measure LF and HF. Measurement and interpretation of HRV was conducted as per guidelines given by the 'Task Force of The European Society of Cardiology and The North American Society of Pacing and Electrophysiology'. ${ }^{4}$

Data analysis was done using RMS Polyrite-D machine and statistical analysis were done by unpaired ' $t$ ' test.

\section{Observations}

Table 1: Mean distribution of age, sex, BMI in the two study groups

\begin{tabular}{|l|c|c|c|c|c|c|}
\hline \multirow{2}{*}{ Parameter } & \multicolumn{2}{|c|}{ Group I (n=30) } & \multicolumn{2}{|c|}{ Group II (n=30) } & t Value & p Value \\
\cline { 2 - 5 } & Mean & SD & Mean & SD & & \\
\hline Age (Yrs) & 32.00 & 4.95 & 31.30 & 5.87 & 0.50 & 0.62 \\
\hline Sex: Male & 20 & & 20 & & 0 & 1 \\
Female & 10 & & 10 & & & \\
\hline BMI & 22.73 & 1.34 & 23.68 & 0.96 & 3.17 & $0.002^{*}$ \\
\hline
\end{tabular}

*p value $<0.05$ considered statistically significant.

Table 2: Comparison of resting pulse, SBP, DBP in the two study groups

\begin{tabular}{|l|c|c|c|c|c|c|}
\hline \multirow{2}{*}{ Resting } & \multicolumn{2}{|c|}{ Group I $(\mathbf{n}=\mathbf{3 0})$} & \multicolumn{2}{c|}{ Group II $(\mathbf{n = 3 0})$} & \multirow{2}{*}{ Value } & \multirow{2}{*}{ P Value } \\
\cline { 2 - 5 } & Mean & SD & Mean & SD & & \\
\hline Pulse rate/min & 72.60 & 3.24 & 77.53 & 7.73 & 3.22 & $0.002^{*}$ \\
\hline SBP $(\mathrm{mmHg})$ & 123.47 & 9.11 & 120.20 & 10.64 & 1.28 & 0.21 \\
\hline DBP $(\mathrm{mmHg})$ & 73.60 & 3.94 & 76.67 & 6.18 & 2.29 & $0.026^{*}$ \\
\hline
\end{tabular}

* $\mathrm{p}$ value $<0.05$ considered statistically significant.

It was observed that there exists a significant statistical difference in the resting pulse and DBP between trained athletes (group I) and sedentary individuals (group II) -Table 2.

Table 3: Comparison of LF, LF(nu), HF, HF(nu), LF/HF, SDNN, RMSSD in two study groups.

\begin{tabular}{|c|c|c|c|c|c|c|}
\hline \multirow[t]{2}{*}{ Resting } & \multicolumn{2}{|c|}{ Group I $(n=30)$} & \multicolumn{2}{|c|}{ Group II $(n=30)$} & \multirow[t]{2}{*}{ t Value } & \multirow[t]{2}{*}{ P Value } \\
\hline & Mean & SD & Mean & SD & & \\
\hline $\mathrm{LF}\left(\mathrm{ms}^{2}\right)$ & 415.43 & 183.91 & 312.70 & 222.02 & 1.95 & $0.056^{*}$ \\
\hline LF (nu) & 77.14 & 6.23 & 77.28 & 4.93 & 0.09 & 0.93 \\
\hline $\mathrm{HF}\left(\mathrm{ms}^{2}\right)$ & 142.07 & 70.18 & 94.71 & 63.59 & 2.74 & $0.009^{*}$ \\
\hline $\mathrm{HF}(\mathrm{nu})$ & 22.83 & 6.21 & 22.46 & 4.61 & 0.26 & 0.79 \\
\hline LF/HF & 3.70 & 1.67 & 3.55 & 0.92 & 0.42 & 0.68 \\
\hline SDNN & 40.86 & 10.95 & 35.93 & 12.87 & 1.60 & 0.12 \\
\hline RMSSD & 29.77 & 11.54 & 20.73 & 8.29 & 3.48 & $0.001^{*}$ \\
\hline
\end{tabular}

*p value $\leq 0.05$ considered statistically significant. 
It was observed that Mean of LF, HF and RMSSD are significantly increased in trained athletes (group I) when compared to sedentary individuals (group II)Table 3.

\section{Discussion}

Globally, the number of cases of lifestyle diseases has increased drastically. The main factor responsible for this significant increase in lifestyle diseases is decrease in physical activity. It has been observed by Lown et al that there exists a significant relationship between the autonomic nervous system and cardiovascular morbidity and mortality. ${ }^{9}$ Autonomic dysfunction to certain extent was noticed in sedentary individuals that in future could pave way to lifestyle diseases. Similar findings were noticed by Noll G et al. ${ }^{10}$

In the present study, as expected significant lower resting pulse rate was seen in trained athletes in comparison to sedentary individuals. This is because, at rest the heart rate is determined by balance between high parasympathetic (Vagal) influence and low sympathetic activity. ${ }^{12}$ Physiological bradycardia in trained athletes is said to be mainly due to long endurance training which increases the "vagal tone". ${ }^{2}$ In our study mean value of Diastolic BP is significantly lower in trained athletes in comparison to sedentary individuals. The findings of our study are in line with the observations made by Sawane MV et al in 2015 which says that physical exercises are known to modulate or 'condition' the autonomic nervous system $^{11}$. The results of our study indicate that regular exercise training reduces resting diastolic BP perhaps due to changes in sympathetic/parasympathetic activation, with alterations in cardiovascular autonomic reflexes.

\section{Heart Rate Variability Measurement at rest ${ }^{13}$ :} Frequency Domain Parameter analysis: Power spectrum by using a mathematical algorithm called the Fourier transformation done. In the present study mean value of LF is increased in trained athletes as compared to sedentary individuals ( $p$ value-0.056). Low frequency reflects autonomic (sympathetic and parasympathetic) factors. Significant increase in HF is seen in trained athletes in comparison to sedentary individuals. Increase in HF band in trained athletes reflects increase in parasympathetic or vagal activity of ANS. No significant difference in LF/HF ratio observed between the two groups.

Time Domain parameter analysis: $\mathrm{N}-\mathrm{N}$ interval of entire recording and statistical measures of variance between NN intervals is taken. Mean value of SDNN is increased in trained athletes than sedentary individuals but is statistically non-significant ( $p>0.05)$.Mean value of RMSSD is increased in trained athlete group and is statistically significant ( $\mathrm{p}$ value $<0.001$ ) It is indicative of increase in parasympathetic activity. Studies conducted by Sawane et al showed similar results and suggested that athletic exercise training program was thought to be a conditioning phenomenon which improved the vagal index. ${ }^{11}$ Panda $\mathrm{K}$ and Krishna $\mathrm{P}^{14}$ conducted a similar study to know the effect of exercise on cardiac autonomic activity. In their study resting heart rate variability was compared in exercising group and non- exercising group. The exercising subjects were found to have a lesser heart rate compared to nonexercising group. In another study, Hottenrott $\mathrm{K}^{15}$ conducted noninvasive test of $\mathrm{HRV}$ for assessment of autonomic changes associated with short-term and long-term endurance exercise training and concluded that regular aerobic training showed a significant improvement of HRV and significant reductions were seen in heart rates at rest and during exercise.

\section{Conclusion}

The results of the study show increase in Heart Rate Variability. Significant increase in High frequency (HF) parameter and RMSSD (Time Domain) parameter of HRV in trained athletes. Decrease in resting heart rate is noted in trained athletes. These indicate parasympathetic (vagal) dominance in trained athletes which is beneficial to cardiovascular system. Therefore this information can be used to make recommendation to the masses to follow exercise regime regularly to help prevent lifestyle diseases.

Prior presentation: Nil

Acknowledgements: Nil

\section{References}

1. Voulgari C, Pagoni S, Vinik A, Poirier P. Exercise improves cardiac autonomic function in obesity and diabetes.Metabolism. 2013 May;62(5):609-21.

2. Prakash S, Meshram S and Ramlekkar U. Athletes, Yogis and individuals with sedentary life styles, Do their lung functions differ? Indian J Physiol Pharmacol. 2007;51(1):76-80.

3. Guyton AC,Hall JE. Guyton and Hall Textbook of Medical Physiology. $12^{\text {th }}$ ed. Indian Reprint:Elsevier 2012;729-39.

4. Task Force Report: Task Force of the European Society of Cardiology and the North American Society of Pacing and Electrophysiology. Heart Rate Variability: standards of measurement, physiological interpretation and clinical use. European Heart Journal. 1996;17:354-81.

5. Yamamoto LM, Lopez RM, Klau JF, Casa DJ, Kraemer WJ, Maresh CM. The effects of resistance training on endurance distance running performance among highly trained runners: a systematic review. J Strength Cond Res. 2008;22(6):2036-44.

6. Neville O, Phillip BS, Geneviève NH, David WD, Charles M. Sedentary Behavior: Emerging Evidence for a 
New Health Risk. Mayo Clin Proc. 2010 Dec;85(12):1138-41.

7. WHO Expert Consultation. Appropriate body-mass index for Asian populations and its implications for policy and intervention strategies. Lancet. 2004;363(9403):157-63.

8. Swash M, Hutchison R. Hutchison's Clinical Methods.

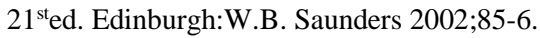

9. Lown B, Verrier RL. Neural activity and ventricular fibrillation. N Engl J Med. 1976;294:1165-70.

10. Noll G, Wenzel RR, Schneider M, Oesch V, Binggeli C, Shaw S, Weidmann P, Lüscher T. Increased activation of sympathetic nervous system and endothelin by mental stress in normotensive offspring of hypertensive parents. Circulation. 1996 Mar;93(5):866-6.9.

11. Sawane MV, Gupta SS. Resting heart rate variability after yogic training and swimming: A prospective randomized comparative trial.Int J Yoga. 2015;8:96-102.

12. Jose $\mathrm{AD}$, Collison $\mathrm{D}$. The normal range and determinants of the intrinsic heart rate in man.Cardiovasc Res.1970 Apr;4(2):160-7.

13. Malik M. Heart Rate Variability. Clin Cardiol. 1990;13:570-6.

14. Panda K, Krishna P. Physical exercise and cardiac autonomic activity in healthy adult men. Indian $\mathrm{J}$ Physiol Pharmacol.2014;58(4):371-5.

15. Hottenrott K, Hoos O, Esperer HD. Heart rate variability and physical exercise. Current status.

Herz. 2006;31(6):544-52.
16. Massin MM, Maeyns K, Withofs N, Ravet F, Gerard P. Circadian rhythm of heart rate and heart rate variability.Archives of Disease in Childhood.2000;83:179-82. 